\title{
Les médias à l'épreuve des crises technologiques
}

\section{Marie-Nöele Sicard}

\section{OpenEdition}

Journals

Édition électronique

URL : http://journals.openedition.org/communicationorganisation/2262

DOI : 10.4000/communicationorganisation.2262

ISSN : 1775-3546

\section{Éditeur}

Presses universitaires de Bordeaux

\section{Édition imprimée}

Date de publication : 1 novembre 1999

ISSN : 1168-5549

\section{Référence électronique}

Marie-Nöele Sicard, «Les médias à l'épreuve des crises technologiques », Communication et organisation [En ligne], 16 | 1999, mis en ligne le 27 mars 2012, consulté le 19 avril 2019. URL : http:// journals.openedition.org/communicationorganisation/2262 ; DOI : 10.4000/ communicationorganisation.2262

Ce document a été généré automatiquement le 19 avril 2019.

(c) Presses universitaires de Bordeaux 


\title{
Les médias à l'épreuve des crises technologiques
}

\author{
Marie-Nöele Sicard
}

1 La plupart des séquences accidentelles résultent bien souvent de la conjonction de phénomènes indépendants créant des combinaisons nouvelles que les concepteurs n'avaient pas prévues par manque d'imagination ou faute d'expérience. Les crises générées par un accident entrent dans une dynamique brutale et déstabilisante qu'on peut utilement éclaircir en s'appliquant à l'étude du champ sémantique du mot «crise » qui est très vaste ${ }^{1} \ldots$

2 Contentons-nous, ici, d'appréhender la crise non pas comme le moment où l'action humaine et l'homme sont des réalités ou des acteurs qu'on peut isoler, définir, expliquer, mais comme le moment suprême de l'interrogation sur le rapport de l'homme avec ses actes.

3 L'intéressant vient de ce que l'état de crise renvoie à la béance de notre savoir, à la prise de conscience aiguë et révélée de façon fulgurante de l'inadéquation au sens, à la raison, en même temps qu'à la nécessité absolue d'agir et de décider.

4 On parle aussi de crise des systèmes ou des organisations lorsque ceux-ci sont menacés dans leur identité sociale et que leur existence même est remise en cause. Dans ce cas, l'état de crise se présente comme une désagrégation de l'identité sociale de l'organisation. La perte du pouvoir associée à cette crise identitaire révèle brutalement l'impossible maîtrise de la complexité d'un monde environnant devenu instable ${ }^{2}$.

5 Quant aux crises technologiques ${ }^{3}$, elles sont des révélateurs qui nous mettent dans la situation de devoir repenser, remettre en œuvre, et surtout reprendre la mesure des technologies. Elles transforment les rapports sociaux et la configuration du lien social, en pointant la difficulté d'articuler la rationalité et le savoir à la peur 4 .

$6 \mathrm{Au}$ moment des crises, les médias sont à la fois les révélateurs grossissants de réalités cachées, les accélérateurs de la dynamique événementielle et les indicateurs critiques des enjeux de pouvoir. 
7 Outils d'urgence et de management ou propagateurs de rumeurs, leur comportement et leur mode de fonctionnement, leur rôle social et politique apparaissent plus clairs lors de crises technologiques, car ils sont à la fois le lieu de la confrontation des pouvoirs en représentation et celui, privilégié, de l'interpellation du politique sur le progrès de la science, de la technologie et de son utilisation.

\section{Représentation médiatique de la crise et déstabilisation sociale}

Les médias construisent alors un certain état de conscience dans la perception du risque et donc dans l'élaboration d'une culture du risque. Car, au moment où ils imposent la transparence et mettent à l'épreuve la qualité du lien social et l'institution politique, la réflexion générale sur le statut de la science et sa capacité à s'établir en instance de contrôle des sociétés modernes vole en éclat, et l'exigence de production d'un savoir immédiat se fait encore plus pressante.

9 Ainsi, par-delà l'observation rationnelle et scientifique des faits inscrits dans le schéma traditionnel de la communication, se produisent des phénomènes qui autorisent à critiquer une conception instrumentale de celle-ci.

Nous émettons l'hypothèse ${ }^{5}$ que les médias d'information "protègent" l'opinion publique du retour à des modes de penser archaïques et génèrent un lien social permettant à la communauté humaine de retrouver une certaine unité donc une possibilité de survivre entre la peur et le savoir. Car, en se constituant pour l'opinion publique comme support de projection des peurs et lieux où se produit la « connection » à la réalité, ils dévoilent la vulnérabilité des sociétés en même temps qu'ils assument leur rôle d'informateurs.

11 La précarité des informations disponibles (le récent exemple de la Dioxine dans les poulets est à ce titre intéressant), la défaillance du discours informatif à un moment où il est particulièrement difficile d'appréhender une réalité technique et scientifique complexe sont des signes de crise; la chronologie des faits et des événements, aussi minutieuse soit-elle, permet, au mieux, d'expliquer quelques-unes des données de l'accident, mais non d'en appréhender la portée. Une sorte de «trou noir» de significations apparaît, qui suscite la peur !

12 L'information est alors transformée par les médias. Dans le système narratif, les faits techniques ne pouvant constituer un moment clé de l'histoire principale, car ils échappent, pour partie, aux compétences des journalistes ${ }^{6}$ et ne sont pas réductibles ni simplifiables, doivent être intégrés dans une histoire qui permet à l'événement d'exister. Celui-ci « appartient » davantage à ses protagonistes qu'aux observateurs. Si, pour ceux-ci il est effectivement difficile d'avoir un accès direct à la réalité, car ils ne disposent pas de la culture technico-scientifique adéquate ${ }^{7} \mathrm{ni}$, souvent, de contacts suffisants ni de temps pour effectuer une enquête approfondie, se faire sa propre idée revient souvent à faire le tour des points de vue différents, la comparaison entre les termes étant prise pour le relief même de la réalité8.

13 La crise accélère les processus et produit un effet de loupe. Elle est aussi et surtout une désynchronisation des temporalités entre les «temps» global, local, politicogestionnaire... 
14 Le temps médiatique se superpose au temps de crise ${ }^{9}$. Cette désynchronisation est accentuée par la perte des repères, des référents, la décontextualisation, la coupure avec l'environnement, le triomphe de l'instant, l'épuisement et non plus l'usure du temps. L'urgence devenant une valeur marchande, la couverture médiatique de l'événement " s'intègre » en quelque sorte à l'événement lui-même.

15 À la fois observateurs d'un événement, générateurs d'une crise qui se développe, prescripteurs du devoir d'informer l'opinion publique en toute liberté, producteurs d'informations soumis à des contraintes bureaucratiques, économiques, indissociables de leur système d'organisation, acteurs sociaux à part entière dans la crise, engagés dans le processus de déstabilisation et le management de l'urgence, voici les médias à la fois témoins extérieurs et divulgateurs de leur vision propre, construite selon leurs valeurs et qui prend pour nous la figure de la réalité.

Souvent sans volonté et/ou possibilité réelle d'investigation des aspects inconscients ou censurés du danger des processus technologiques, mais volontiers propagandistes des peurs, ils structurent l'espace public, le lieu privilégié du débat démocratique. Et pourtant, à y regarder de plus près, on peut trouver dans cette multiplicité contradictoire de fonctions et de rôles des paradigmes unificateurs.

18 La substitution des objets construits ${ }^{10}$ au " réel » permet de conférer un pseudo "sens" aux événements néfastes porteurs de danger et de donner une figure à l'angoisse. Les médias en outre, constituent par l'artifice des faits de séries une "mémoire des crises » où, à travers la narration de l'histoire, c'est le passage du réel à l'imaginaire qui est assuré. Ils trouvent là une façon de faire partager une même expérience aux acteurs sociaux et fondent ainsi une sorte de renouement social.

20 Si la sécurité s'acquiert au terme d'une objectivation du monde gérée dans des catégories rationnelles, le traitement de la perception du risque permet d'articuler la peur et le savoir. En ce sens, les médias sont effectivement le seul lieu ou se produisent nos représentations et où la perception du risque majeur et l'information du citoyen trouvent dans un discours « en trompe l'œil » une sorte de scénographie, le moyen d'exister ${ }^{11}$.

21 Leur pouvoir extrême est dû à leur capacité de fédérer les peurs tout en attirant une large audience, à leur aptitude à modéliser la réalité et donc à rétablir une causalité dans le déferlement des crises technologiques et à inventer une histoire des crises. Leur faiblesse extrême demeure l'ambiguïté d'une vocation à assurer un libre espace de circulation du discours, vital, lorsqu'il s'agit de risques technologiques et d'être en même temps contraints de gérer « un produit » : l'information dans un système de valeurs spécifiques. D'un côté assurer et conforter une maîtrise gestionnaire contrainte par l'urgence, de l'autre, construire un espace symbolique de liberté, lieu de représentation du citoyen, contribuer à l'édification d'une mémoire.

Peut-être est-ce en fabricant une information qui ne renvoie pas toujours à l'événement mais à la promotion de l'information pour elle-même comme événement, et en activant le champ communicationnel que cette ambiguïté constitutive peut perdurer. Elle produit avant tout un «faire social» libéré de la contrainte immédiate de générer du sens à condition de proliférer dans l'instant, mais elle doit aussi et en même temps, offrir le terrain propice à la projection des angoisses et au partage des symboles dans l'imaginaire. 


\section{Système complexe et temporalités}

23 font éclater la temporalité par l'obsession, la surchauffe, la sacralisation du présent. Ce temps, que nous appellerons chronoscopique, opère une sorte de transmutation de la profondeur de champ et donc de l'épaisseur optique de l'environnement humain, il se définit en termes de visibilité, de sous-exposition, ou de surexposition. L'impératif de survisibilité événementielle qui entre dans la logique du traitement médiatique, escamote souvent la trajectoire diachronique événementielle et perturbe la compréhension et l'interprétation de la crise.

Si l'accident moderne est un "non-événement ", car il est précisément ce qui ne doit jamais arriver, ses effets demeurent souvent invisibles, incertains, diffus, décalés dans le temps et dans tous les cas difficiles à objectiver.

"L'épreuve " devient donc événement quand elle est médiatisée, "couverte ", l'événementialité de l'événement étant inséparable du média qui le prend en charge.

Sur le devant de la scène apparaît la rupture de la croyance en un contrôle généralisé et resurgit avec violence l'interrogation sociétale sur les buts de l'action organisée en vue de l'utilité, de l'efficacité technique.

Cette blessure narcissique de la société qui échoit, se situe à l'intérieur même du discours technico-politique tenu par les médias. Le travail de recodification des médias d'information peut alors être considéré comme une forme particulière d'anamorphose de la réalité qui fonctionne comme un système complexe. Dans ce système s'exposent, coexistent, évoluent des combinaisons narratives qui modifient la perception des risques et accroissent la vulnérabilité sociale.

De fait, tout en conservant la logique de leurs valeurs et le contrôle de leurs procédures, les médias, à travers leur conquête et leur prise en charge des publics contribuent à faire évoluer leurs perceptions. Celles-ci, à leur tour, rétroagissent sur la formalisation des codes du discours médiatique.

conjointement des formes d'ordre et de désordre, une réalité elle-même performée par les attentes des publics et leurs perceptions des modalités discursives.

La survenue de la crise technologique ajoute encore une dimension au système complexe. Du point de vue d'une sémiotique des médias, elle est définie comme la rupture d'une continuité narrative, comme une phase d'indécision, de désordre devant laquelle s'ouvrent une multiplicité d'options narratives qui tressent un grand nombre d'événements possibles dans l'économie générale d'une chronologie ${ }^{12}$.

Dès lors, ce brouillage des temps et cette sacralisation du présent laissent place à une relation inédite entre le présent et le passé, aux dépens d'un avenir ressenti comme de plus en plus imprévisible et menaçant.

Ainsi, à défaut d'assurance sur le futur et de solidarité entre le passé et l'avenir, émerge une autre forme de solidarité entre le présent et la mémoire.

L'acculturation, la mise en patrimoine des crises survenues à la suite de catastrophes comme Seveso, Bhopal, Challenger, Tchernobyl, Bâle, est issue de leur médiatisation à travers le monde dans différentes cultures ${ }^{13}$. 
Pour les publics, il devient plus sécurisant de vivre au rythme des illusions d'un présent " surchauffé » au contact d'une actualité brûlante qui ne s'arrête jamais, que de prendre la réelle mesure et d'intérioriser un événement catastrophique, à moins de le sublimer en œuvre d'art ${ }^{14}$. Cet enchevêtrement des modalités temporelles qui conditionne les stratégies discursives fait apparaître clairement la façon dont l'événement se transforme, comment la trajectoire performatrice fait basculer les publics qui n'accèdent pas à une représentation de l'événement mais le « suivent », ou " y croient », éventuellement.

Car la loi est celle du présent qui n'enchaîne pas avec les autres temps mais se succède à lui-même sous la forme d'un autre présent, le procès n'étant fait que du surgissement perpétuel d'une différence à l'horizon. La crise a la propriété d'introduire entre elle et nous une tension qui génère une attente, un désir qui sera comblé par la mise en présence de l'événement dans l'actualité.

C'est alors que la vulnérabilité de la société, c'est-à-dire sa perception des risques, augmente d'autant plus que le temps, la maturation manquent pour construire des opinions réfléchies et fondées.

La nouvelle relation au temps instaurée par les médias accentue encore cette fragilité des publics.

Ceux-ci voient consacrée l'autonomisation de deux instances : l'avenir rendu tout entier à son imprévisibilité, puisque la démonstration est faite qu'aucune catastrophe n'est évitable et du même coup, obsédant, le passé détaché de la cohérence organisatrice d'une histoire et devenu tout entier patrimonial ${ }^{15}$.

En situation de crise, la temporalité traditionnelle est déstructurée.

Les médias font apparaître qu'il ne peut y avoir de conscience historique des crises technologiques car aucune continuité ne peut être établie entre les différents événements, chacun étant en soi le signe d'une rupture unique.

En outre, les médias enferment chaque événement dans un présent sacralisé qui dénie l'existence d'une chaîne causale qui relierait le passé et le présent.

La reprise par le mémorial est une façon de renouer avec la continuité.

«Le lieu de mémoire est l'unité significative d'ordre matériel ou idéel dont la volonté des hommes ou le travail du temps a fait un élément symbolique d'une quelconque communauté » (Nora, 1993).

3 À défaut de se donner une assurance sur le futur, les crises technologiques passées deviennent le patrimoine culturel, les traces-vestigia que laissent notre société. Celles-ci constituent notre seule assurance sur le passé sans pour autant pouvoir garantir le moins du monde l'avenir.

44 Si cette distorsion de la temporalité influe souterrainement sur l'inquiétude actuelle, c'est parce que les médias opèrent la double démarche d'immortaliser dans le patrimoine et de sacraliser dans le présent, autrement dit d'évacuer la continuité temporelle de la réalité qui, en situation de crise est ingérable.

\section{Impératif chronoscopique et crise}

45 La crise se développe dans un temps diégétique qui obéit à un ordre de succession chronologique (elle a un début, un milieu et une fin), alors que conjointement la 
couverture médiatique en donne une vision «chronoscopique » qui privilégie l'enjeu de visibilité et les valeurs de sur ou sous exposition.

Les médias sont à la fois coproducteurs et inducteurs de la chaîne événementielle et promoteurs d'un temps qui s'expose et, par là, qui risque de faire perdre aux événements toute densité en donnant à voir des figures hypertrophiées sans épaisseur référentielle ou contextuelle. L'analyse des titres du Monde dans la crise de la vache folle entre le 22 mars et le 26 avril 1996 est significative ${ }^{16}$.

Cadrage et collage, pratiques journalistiques ordinaires, produisent des effets de monstration. Le cadrage opère à la fois une coupure et une focalisation en isolant un fragment de l'expérience et en le séparant de son contexte; il rend difficile la prise en compte et la vision d'ensemble d'une situation, et renforce l'effet de crise.

Le collage qui sous-tend la constitution d'un texte fragmentaire, cette nouvelle totalité née de morceaux, de déchets d'actualité, joue avec les changements d'échelle, la relativisation de la partie et du tout. La juxtaposition d'états fragmentaires de points de vue constellés dessine un espace médiatique qui donne l'illusion de la mobilité de la parole.

49 Pour être visible l'événement doit être envisagé dans la dynamique inépuisable d'appréhensions multiples.

Ce temps que nous appelons «chronoscopique » opère une sorte de transmutation de la profondeur de champ et donc de l'épaisseur optique de l'environnement humain. Cette esthétique du dévoilement excessif obère la réalité ; à force de transparence voulue, c'est souvent l'obscurité qui domine ; l'impératif de survisibilité événementielle escamote souvent la trajectoire diachronique événementielle et perturbe la compréhension et l'interprétation des événements et par voie de conséquence intensifie la crise.

51 En outre, l'interférence de temporalités différentes : celle de la crise et celle des médias crée des zones de turbulence et d'opacité. La surchauffe du présent entretenue par les médias accuse la perte du sentiment, de ce qu'on pourrait appeler "l'expérience historique ».

Notre expérience moderne se constitue plutôt comme une succession de présents aussitôt ouverts que recouverts. La machine médiatique en produisant du présent affaiblit toutes les formes de représentation et favorise une mutation du rapport des publics au temps.

Les médias ne peuvent constituer « une histoire » des catastrophes ou des crises en raison du brouillage des temporalités et compte tenu aussi de la nature des événements qui, par essence, «échoient », c'est-à-dire arrivent sans qu'on puisse déterminer de véritables liens de causalité et prévoir les faits.

La mise à distance de la crise s'exprime par la «trace » qu'en ont laissée les médias. Car, par la production de signes au quotidien qu'ils donnent à consommer en continu, et l'éphémérité maintenue en scène par leurs contraintes fonctionnelles, ils interdisent toute intériorisation de la crise et donc tout travail de deuil des publics.

$55 \mathrm{Au}$ mieux, le temps réinvesti dans une mémoire patrimoniale leur permet de se situer, mais ils demeurent des passagers "embarqués " par un média qui évoluent d'un échangeur à l'autre et non des voyageurs convoqués à un carrefour pour une véritable rencontre. 


\section{Conclusion}

Quelques phénomènes nous semblent importants à souligner : en particulier le fait que le temps médiatique fonde une conscience sociale des crises et constitue un patrimoine mémoriel réactivé lors du surgissement d'une nouvelle crise (la vache folle après le sang contaminé $)^{17}$.

$$
\begin{aligned}
& \text { que les mécanismes d'appropriation d'un «s } \\
& \text { médiatique n'est pas celui de la connaissance. }
\end{aligned}
$$
l'utopie de la transparence implique que tout est dicible. social est sans fin.

Le surcodage, le surencadrement, la surabondance de signes rend vulnérable à la fureur et au bruit. Le vrai cède sa place au vraisemblable; le système médiatique risque de céder à la tyrannie de l'économie et de l'urgence, le souci de l'effet générant l'ère du soupçon...

Mais gageons qu'il nous appartient de refuser que le système médiatique en situation de crise produise une sorte de façon d'être au monde instrumentalisée qui nous dépossède de notre citoyenneté !

\section{BIBLIOGRAPHIE}

LAGADEC Patrick, États d'urgence, Paris, Seuil, 1988. 
LEGOFF Jacques, Faire de l'histoire, Paris, Gallimard 1974.

NoRA Pierre, Les lieux de mémoire, Paris, Gallimard, 1993, t. 3.

SALOMON Jean-Jacques, « Maîtrise sociale de la technologie : l'enjeu démocratique », in Futuribles,

Paris, septembre 1991.

SCANLON Joseph, The role of the media in crisis situation, Ottawa, Carleton University, 1988.

SICARD Marie-Noële, Entre médias et crises technologiques, les enjeux communicationnels, Lille,

Septentrion Presses universitaires, 1998.

THEYs Jacques, La société vulnérable, Paris, Presses de l'ENS, 1987.

\section{NOTES}

1. Voir notamment les articles de E. Morin «Pour une crisiologie » et J. Freund « Observations sur deux catégories de dynamiques polémogènes ; de la crise au conflit » in Communications, $\mathrm{n}^{\circ} 25$, Paris, Seuil, 1976.

2. J. Habermas, « Raison et légitimité », Paris, Payot, 1988, p 12.

3. Sur ce point, voir P. Lagadec, « La gestion des crises », Paris, Mac Graw Hill, 1991, p. 43 sq.

4. Denis Duclos, «La peur et le savoir », Paris, La Découverte, 1989.

5. Cette hypothèse est fondée sur l'analyse d'un corpus d'articles concernant la crise de Sandoz Schweizerhalle de 1986 (M.-N. Sicard, « Les médias en situation de crise techologique »)HDR soutenue à l'Université Paris X Nanterre, 1994.

6. cf L'analyse de R.B. Irvine à propos de l'accident de Bhôpal «When you are the headline managing a major news story », Homewood, Illinois, Dow-Jones 1997.

7. E. Quarantelli, "A preliminary statement on the differente world of science and mass communication : implications for information flow between them ", in Publications of The Disaster Research Center, University of Delaware, USA, October 1990.

8. M. Stephens, W. Edison, "News coverage of issues during the accident of TMI ", journalism Quartely 59, 1982, pp. 199-204.

9. cf. l'analyse éclairante de P. Lagadec dans « la gestion des crises » cf supra.

10. Voir Eliseo Veron, "Construire l'événement », Paris, Éditions de Minuit, 1981.

11. P.M. Sandman, "Explaining risk to non experts", conference on global disasters and international information flow, the Annenberg School of Communication, Washington DC, 1986.

12. M.-N. Sicard, «Entre médias et crises technologiques », Lille, PU Septentrion, 1998, p. 131 sq.

13. F. Choay, «L'allégorie du patrimoine », Seuil, Paris, 1992, p. 147.

14. Cf La photographie du sarcophage de Tchernobyl par Louis Jammes.

15. B. Ollivier, «La puissance technologique et la fragilité sociale », Futuribles $n^{\circ} 156$, juillet-août 1991.

16. M.-N. Sicard, «Pratiques journalistiques et enjeux de la communication scientifique et technique » in Hermès Communication et politique «Sciences et médias » n² 21 Paris, 1997.

17. Actes du Séminaire du Programme "Risques collectifs et situation de crises », CNRS, février 1996.

18. P. Mann, «Théories de la mobilisation », Actes du Séminaire du programme " Risques collectifs et situation de crises », CNRS, décembre 1996. 


\section{RÉSUMÉS}

La situation paroxystique générée par un grand accident technologique offre une opportunité pour étudier le comportement et le fonctionnement des médias d'information. Le système médiatique opère comme un système complexe qui transforme son objet, la crise technologique, en une nouvelle réalité politique et sociale. Les discours de mise en sens, de mise en scène, «connectent» l'opinion publique à un espace événementiel qui entrave sa faculté de représentation du risque et accroît ainsi sa vulnérabilité.

The extreme situation generated by a technological disaster offers a unique opportunity to study the behaviour and functionning of information médias. The media System is a complex System which transforms its own subject, the technological crisis, into a new political and social reality. The substance and the form by which the information is trans-mitted and the way that the public opinion is connected to such space of information prevents the recipient from really understanding the risk of technological disaster, thus rendering them more vulnerable.

\section{AUTEUR}

\section{MARIE-NÖELE SICARD}

Marie-Noële Sicard est Maître de Conférences en Sciences de l'information et de la communication à l'Université de Technologie de Compiègne, et habilitée à diriger les recherches. Ses enseignements et ses recherches portent sur les rapports entre les médias d'information et la société et en particulier sur la problématique de la communication de la science et de la technologie dans la presse d'information et à la télévision. 